This item was submitted to Loughborough's Research Repository by the author.

Items in Figshare are protected by copyright, with all rights reserved, unless otherwise indicated.

\title{
Increased serum adiponectin concentrations in amenorrheic physically active women are associated with impaired bone health but not with estrogen exposure.
}

\section{PLEASE CITE THE PUBLISHED VERSION}

http://dx.doi.org/10.1016/j.bone.2010.12.018

\section{PUBLISHER}

(C) Elsevier

\section{VERSION}

AM (Accepted Manuscript)

\section{PUBLISHER STATEMENT}

This work is made available according to the conditions of the Creative Commons Attribution-NonCommercialNoDerivatives 4.0 International (CC BY-NC-ND 4.0) licence. Full details of this licence are available at: https://creativecommons.org/licenses/by-nc-nd/4.0/

\section{LICENCE}

CC BY-NC-ND 4.0

\section{REPOSITORY RECORD}

O'Donnell, Emma, and Mary Jane De Souza. 2019. "Increased Serum Adiponectin Concentrations in Amenorrheic Physically Active Women Are Associated with Impaired Bone Health but Not with Estrogen Exposure.". figshare. https://hdl.handle.net/2134/20630. 


\section{Increased Serum Adiponectin Concentrations in Amenorrheic Physically Active Women}

2 are Associated with Impaired Bone Health but not with Estrogen Exposure

4 Emma O’Donnell ${ }^{1,2}$ and Mary Jane De Souza ${ }^{3}$

$6 \quad{ }^{1}$ Cardiovascular Research Laboratory, ${ }^{2}$ Women’s Exercise and Bone Health Laboratory,

7 Department of Exercise Sciences, University of Toronto, Toronto, Ontario, M5S 2W6, Canada;

$8{ }^{3}$ Women's Health and Exercise Laboratory, Department of Kinesiology, Pennsylvania State

9 University, 104 Noll Laboratory, University Park, PA, 16803 ,USA.

12 Corresponding Author:

13 Mary Jane De Souza, Ph.D., FACSM, Women’s Health and Exercise Laboratory, Department of

14 Kinesiology, The Pennsylvannia State University, 104 Noll Laboratory, University Park, PA

15 16803. Tel: 814-863-0045, Fax: 814-865-4602, Email: mjd34@psu.edu

16

17 Key terms: adipokines, adipose tissue, amenorrhea, bone, exercise

18

19 Abbreviated Title: Adiponectin in Amenorrheic Athletes 


\section{Abstract}

3 Background: The role of adiponectin in mediating gonadal status and bone health in weight-

4 stable healthy adult female athletes with secondary amenorrhea has not yet been described.

5 Methods: Using a prospective observational study, age matched premenopausal women were

6 studied, including: 1 ) sedentary ovulatory women (SedOv; $n=10$ ), 2) exercising ovulatory women

7 (ExOv; $n=15)$, and 3) exercising amenorrheic women (ExAmen; $n=9)$. Primary outcome

8 measures included serum total adiponectin and daily urinary estrogen (E1G) levels, expressed as

9 area under the curve (AUC), body fat distribution, and bone mineral density (BMD). Serum

10 leptin, ghrelin, total triiodothyronine (TT3), insulin, and resting energy expenditure (REE) were

11 also determined.

12 Results: The women in this study did not differ in age (25.3 \pm 1.4 years; mean \pm SEM), height

$13(164 \pm 1 \mathrm{~cm})$, weight $(57.7 \pm 1.0 \mathrm{~kg})$ and BMI $\left(21.5 \pm 0.4 \mathrm{~kg} / \mathrm{m}^{2}\right)$. Exercising women had a higher fat

14 free mass (FFM), lower fat mass (FM) and lower serum leptin concentrations $(p<0.05)$ compared

15 to sedentary women. Adiponectin and ghrelin levels were higher $(p<0.05)$, and TT3 $(p=0.019)$,

16 urinary E1G AUC ( $p=0.002)$ lower in ExAmen compared with ExOv and SedOv. Total and L1-

17 L4 BMD were lower $\left(\mathrm{p}^{<0.05}\right)$ in ExAmen compared with ExOv. Stepwise linear regression

18 identified trunkal FM as the strongest predictor of log adiponectin adjusted for FM $(F=23.54$,

$19 p<0.001)$. L1-L4 BMD was predicted by log adiponectin and E1G AUC $(F=9.856, p=0.045)$.

20 Total BMD was predicted by $\log$ adiponectin $(F=7.948, p=0.009)$. TT3 was the strongest

21 predictor of E1G AUC $(F=9.885, p=0.004)$.

22 Conclusions: Hypoestrogenic adult female athletes with secondary amenorrhea demonstrate

23 elevated circulating adiponectin relative to FM in association with impaired bone health. Estrogen

24 exposure was predicted by TT3, but not adiponectin. These findings suggest that nutritionally

25 regulated hormones may mediate gonadal status, and that adiponectin and estrogen, either 
1 independently or in combination, may mediate bone health in adult amenorrheic physically active 2 women. 


\section{1. INTRODUCTION}

In exercising women, energy deficiency, a consequence of inadequate caloric intake

3 relative to exercise energy expenditure, has been proposed as the causal mechanism of menstrual

4 disturbances [1, 2]. While not all exercising women experience symptomatic menstrual

5 disturbances, between 2-44 \% of female athletes have reported amenorrhea, the most severe

6 menstrual disorder [3, 4]. Secondary to inadequate caloric intake, energy deficient amenorrheic

7 athletes demonstrate hypoestrogenemia and a hypometabolic state, including suppressed

8 concentrations of glucose, leptin, insulin, and total triiodothyronine (TT3) and elevated levels of

9 ghrelin and peptide YY [5-10]. In adolescent amenorrheic athletes, elevated ghrelin and reduced

10 leptin concentrations predict suppressed levels of gonadal steroids [8]. It is not known if these, or

11 other factors, such as adiponectin, similarly predict gonadal status in adult amenorrheic athletes.

12 Adiponectin is an adipose tissue-specific secretory protein that is expressed exclusively

13 in differentiated adipocytes [11]. In contrast to other adipokines, such as tumor-necrosis factor

14 and interleukin-6, which are upregulated with increasing adiposity, adiponectin concentrations

15 correlate negatively with obesity and insulin resistance [12, 13]. Conversely, adiponectin

16 concentrations have been reported to correlate both positively [14] and negatively [15] with low

17 body weight, i.e., anorexia nervosa. Reasons for discrepancy in anorexia nervosa are unclear, but

18 may be related, in part to body-fat distribution. For example, recent in vitro evidence

19 demonstrates that the rate of adiponectin secretion is approximately threefold higher from visceral

20 compared with subcutaneous fat, suggesting that distribution of body fat rather than total fat

21 amount may be important [16]. Examination of possible relationships between fat-deposition site

22 and circulating adiponectin concentrations in premenopausal eumenorrheic and amenorrheic

23 women has not yet been reported.

24 The regulatory role and consequences of altered adiponectin metabolism are not yet well

25 described. Studies indicate, however, that metabolic rate may be increased and bone health

26 impaired by hypo- and hyper-adiponectinemia, respectively [14, 17]. Several studies have also 
1 demonstrated that women have higher adiponectin levels compared to men, independent of body

2 fat mass or body fat distribution [13, 18], suggesting that circulating gonadal steroids may affect

3 adiponectin secretion [19]. To date, however, there have been no reports describing whether

4 estrogen exposure, as assessed by daily urinary analysis of ovarian steroids over time, is mediated

5 by nutritionally regulated hormones, such as adiponectin, in young adult physically active

6 amenorrheic women. Such assessment may increase the ability to detect estrogenic associations,

7 when present, compared with one-time sample serum measures of estradiol.

8 The objectives of the current study were threefold. In eumenorrheic ovulatory athletes

9 and amenorrheic athletes, to explore: 1) the relationship between adiponectin concentrations and

10 gonadal status (i.e., estrogen exposure) as assessed via daily urinary measures over time; 2) the

11 relationship between adiponectin and nutritionally mediated factors known to be altered in

12 response to energy deficiency, including TT3 and resting energy expenditure; and 3) the

13 associations between adiponectin, body composition, body fat distribution and bone health. We

14 hypothesized that compared to estrogen replete women, hypoestrogenic physically active adult

15 premenopausal women with energy deficiency associated amenorrhea (EDAA) would

16 demonstrate elevated serum adiponectin concentrations, and that these concentrations would

17 predict gonadal status and bone health. We further postulated that adiponectin concentrations

18 would be negatively associated with nutritionally mediated factors known to be altered in

19 response to energy deficiency. Finally, we hypothesized that central (i.e., visceral) adipose mass

20 would demonstrate stronger associations with adiponectin compared with peripheral (i.e.,

21 subcutaneous) adipose deposition.

\section{2. METHODOLOGY}

\section{$24 \quad 2.1$ Participants}

25 Participants were recruited by posters. Physical activity status was required to have been 26 consistent for the previous 6 months. Eligibility criteria for the study included: 1) age 18 to 35 
1 yrs; 2) good health determined by a medical exam; 3) no chronic illness, including

2 hyperprolactinemia, polycystic ovarian syndrome, and thyroid disease; 4) stable self reported

3 menstrual status (i.e., same menstrual status) over the preceding 3 months, with menstruating

4 women having cycle lengths between 25-35 days, and athletic women with secondary

5 amenorrhea having not menstruated for at least 90 consecutive days [4]; 5) non-smoking; 6) not

6 currently dieting and weight stable for the preceding 3 months, as determined by self-report; 7)

7 absence of hormonal therapy for at least 12 months; 8) no history or current clinical diagnosis of

8 eating disorders and 9) no other contraindications that would preclude participation in the study.

9 The study was approved by the institutional committee on human research by the Ethics Review

10 Board at the University of Toronto, and confirmed to the standards set by the latest revision of the

11 Declaration of Helsinki. All volunteers signed an approved informed consent document.

\section{$13 \quad 2.2$ Experimental Design}

14 We originally conducted a prospective observational study on a rolling basis over 2-3

15 years to examine relationships between physical activity, metabolism, cardiovascular health and

16 reproductive function. Fifty-two women completed the entire study, with 34 of these 52 being

17 included in the current post-hoc study. The relationship between estrogen exposure and

18 numerous metabolic and nutritionally regulated hormones on cardiovascular function in a subset

19 of these women have previously been described by our laboratory [6, 20]. However, the potential

20 for nutritionally regulated hormones, specifically adiponectin, and indices of metabolic status,

21 namely TT3 and REE, to predict gonadal status has not been previously reported by our group.

\section{$23 \quad 2.3$ Observational Time Periods}

24 Menstruating women were monitored for 2 to 3 consecutive menstrual cycles, and 25 amenorrheic women were monitored for 2 to 3 consecutive 30-day monitoring periods. All

26 measures, except urinary measures which were assessed daily, were obtained during the early 
1 follicular phase (days 2-6) across two-to three menstrual cycles for menstruating women, and

2 during days 1-6 of each 30-day monitoring period for amenorrheic women. The mean of these

3 measures were used in statistical analyses.

$5 \quad 2.4$ Exercise and Menstrual Status

6 Exercise status was defined as “sedentary” when purposeful exercise was less than 2

7 hours per week and “exercising” when purposeful exercise was more than 2 hours per week [21].

8 Purposeful exercise, defined as exercise that elicited a heart rate (HR) greater than $55 \%$ of

9 maximal HR (220 minus age) for 3 minutes or more, was documented in exercise logs [6]. HR

10 was determined by the subject counting heart beats during a 10 second period of carotid artery

11 palpation after each exercise bout. In conjunction with the hours of exercise activity criterion, we

12 also utilized a $\mathrm{VO}_{2}$ max of $<40 \mathrm{ml} / \mathrm{kg} / \mathrm{min}$ to reflect sedentary status and $40 \mathrm{ml} / \mathrm{kg} / \mathrm{min}$ or greater

13 to reflect exercising status consistent with published data of this parameter [22].

14 Menstrual status was determined from daily first morning void urine samples collected by

15 all participants for the duration of the study period. Urine samples were assayed for luteinizing

16 hormone (LH), pregnanediol 3-glucuronide (PdG), and estrone 3-glucuronide (E1G) to assess

17 ovulatory status and estrogen exposure. Our group has previously detailed the criteria for

18 detection of positive ovulation and menstrual status [6].

\section{$20 \quad 2.5$ Estrogen Exposure}

21 Calculation of estrogen exposure over time has been described, in detail, elsewhere [6].

22 Briefly, using daily urinary estrogen levels, estrogen exposure over time was calculated by the

23 trapezoidal area under the curve method across two to three menstrual cycles for menstruating

24 women, and across two to three 30-day monitoring periods for amenorrheic women. 


\section{$1 \quad 2.6$ Study Groups}

Three groupings were retrospectively established based on exercise and menstrual status:

3 1) sedentary women with ovulatory menstrual cycles (SedOv; $n=10$ ), 2) exercising women with

4 ovulatory menstrual cycles (ExOv; $n=15)$, and 3) exercising women with amenorrhea, defined as

5 cessation of menses for $>90 \mathrm{~d}$ (ExAmen; $n=9)$ [23].

$7 \quad 2.7$ Anthropometric Measures

8 Average total body mass was determined from weekly measures to the nearest $0.1 \mathrm{~kg}$ on

9 a physician’s balance scale (Detecto, Webb City, MO). Height was measured to the nearest 1.0

$10 \mathrm{~cm}$ at the beginning of the study period. Body mass index (BMI) was calculated $\left(\mathrm{kg} / \mathrm{m}^{2}\right)$.

\section{$12 \quad 2.8$ Body Composition and Bone Mineral Density}

13 Dual-energy x-ray absorptiometry (DXA) was utilized to determine body composition

14 and bone mineral density (BMD) once during the study by a trained technician (Prodigy, General

15 Electric Lunar Corporation, Madison, WI, enCORE 2002 software, version 6.50.069). Whole

16 body and lumbar (L1-L4) BMD $\left(\mathrm{g} / \mathrm{cm}^{2}\right)$ and Z-scores were determined. Central fat mass (kg) was

17 determined from the fat mass measured in the trunk, and peripheral fat mass was determined as

18 the sum of fat mass measured in the arms and legs. BMD $\left(\mathrm{g} / \mathrm{cm}^{2}\right)$ was determined at the spine

19 (L1-L4) and for total body by an ISCD certified operator. A 28 subject precision study was

20 performed in premenopausal women and precision was 0.6 and $0.7 \%$ at the total body and

21 lumbar spine, respectively. The DXA scanner has a precision of $<1 \%$ coefficient of variation for

22 body composition measurements.

\section{$24 \quad 2.9$ Resting Energy Expenditure}

25 Using the Weir equation [24], REE (kcal day ${ }^{-1}$ ) was determined by indirect calorimetry

26 with a ventilated hood system (SensorMedics Vmax Series, Yorba Linda, CA). After a 45 minute 
1 supine rest period, REE measures were taken for 30 minutes between 0830 and 1100 . Oxygen

2 consumption $\left(\mathrm{VO}_{2} ; \mathrm{mL} \mathrm{min}^{-1}\right)$ and carbon dioxide production $\left(\mathrm{VCO}_{2} ; \mathrm{mL} \mathrm{min}^{-1}\right)$ were measured

3 every 20-seconds during REE measurement. To calculate REE, data for $\mathrm{VO}_{2}$ and $\mathrm{VCO}_{2}$ were

4 only used if steady state was attained for a minimum of 10 minutes, and respiratory quotient

5 values did not varying by more than $10 \%$. REE adjusted for fat free mass (FFM) was calculated

6 to adjust metabolic rate for metabolically active tissue (i.e., muscle).

$8 \quad 2.10$ Peak Aerobic Capacity

$9 \quad \mathrm{VO}_{2}$ peak was measured once by a progressive treadmill test to volitional exhaustion.

10 Expired gases were collected continuously to measure inspired air volumes and to analyze breath-

11 by-breath samples (Moxus Modular $\mathrm{VO}_{2}$ System, Applied Electrochemistry Inc., Pittsburgh, PA).

\section{$13 \quad 2.11$ Serum Measures}

14 Serum was analyzed for total adiponectin, leptin, total ghrelin, total triiodothyronine

15 (TT3) and insulin. Eight-hour fasted blood samples were collected between 0730 and $1000 \mathrm{hr}$.

16 Serum samples were immediately stored at $-80^{\circ} \mathrm{C}$ until analyses were run. Total adiponection

17 (i.e., low, medium, and high molecular weight) was analyzed using an enzyme immunoassay

18 technique (Quantikine Assay Kit, DRP300, R\&D Systems Inc., Minneapolis, MN). Sensitivity

19 was $0.246 \mathrm{ng} / \mathrm{mL}$. Leptin was analyzed using a direct sandwich enzyme-linked immunosorbent

20 assay (ELISA, Linco Research, Inc., St. Charles, MO). Analytical sensitivity was 0.5 ng/mL.

21 Total serum ghrelin was analyzed using radioimmunoassay techniques (Linco research Inc., St

22 Charles, MO). Analytical sensitivity of the ghrelin assay was $2.97 \mathrm{pmol} \mathrm{L}^{-1}$. Serum insulin and

23 TT3 were analyzed using a chemiluminescence-based immunoassay analyzer (Immulite,

24 Diagnostics Products Corporation, Los Angeles, CA). Analytical sensitivity for the insulin assay

25 was $13.89 \mathrm{pmol} \mathrm{L}^{-1}$, and for the TT3 assay was $0.54 \mathrm{nmol} \mathrm{L}{ }^{-1}$. All inter- and intra-assay

26 coefficients of variation have previously been reported by our group [6, 20]. 


\section{$2 \quad 2.12$ Urinary Measures}

3 Measures of daily urinary metabolites were determined using microtiter plate competitive

4 enzyme immunoassays to detect pregnanediol 3-glucuronide (PdG), and estrone 3-glucuronide

5 (E1G). Detailed methods for these immunoassays have been described previously [6, 20]. The

6 inter-assay coefficients of variation for high and low internal controls were $14.7 \%$ and $13.1 \%$ for

$7 \quad$ E1G and $15.68 \%$ and $17.7 \%$ for PdG. Urine samples were corrected for specific gravity using a

8 hand refractometer (NSG Precision Cells, Inc., Farmingdale, NY) to account for hydration status.

9 Urinary LH, assessed in ovulatory women only, was determined by double antibody

10 radioimmunoassay (Diagnostic Products Corp., Los Angeles, CA). The sensitivity of the LH

11 assay was $0.6 \mathrm{mIU} \mathrm{L}^{-1}$. The intra-assay and inter-assay coefficients of variation were $1.6 \%$ and

$12 \quad 7.1 \%$, respectively.

\section{$14 \quad 2.13$ Statistics}

15 Data screening, conducted prior to statistical analysis included outlier detection, and

16 examination of variable distributions within each of the three groups for normality. No outliers

17 were detected, but adiponectin, leptin and urinary progesterone were determined to be positively

18 skewed and were log transformed to approximate normal distribution. All other data sets were

19 normally distributed. Comparison of data between all three groups were analyzed using one-way

20 ANOVA, and when a significant main (fixed) effect was observed, the least significant squares

21 was used to determine where the significant differences existed. Multiple comparisons were

22 adjusted by using Bonferroni methods. Comparisons between two groups were analyzed using

23 independent samples $t$-test. Using pooled data, Pearson's bivariate correlational analyses were

24 used to determine significant linear independent associations between adiponectin, estrogen

25 exposure, and all other variables of interest. Running separate analyses, mixed model linear

26 regression using stepwise methods ( $P=0.15$ for entry, and $P=0.20$ to leave the model) were used 
1 to explore predictors of gonadal status, adiponectin levels and bone health. Variables included in

2 the models were hormones and parameters of body composition of interest to the current study, as

3 well as those that have been previously shown by others to be associated with each dependent

4 variable of interest, regardless of whether significant bivariate correlations were observed

5 between these variables and the dependent variable. This method of inclusion was selected to

6 account for potential confounding effects of various variables and to rule out the masking of

7 associations of various independent variables with the dependent variable because of

8 confounders. Data were analyzed using packaged software (SPSS version 12.0; SPSS Inc.,

9 Chicago, IL). A significance level of $P<0.05$ was used to detect the differences for statistical

10 procedures. The mean of 2-3 values for serum adiponectin, leptin, ghrelin, TT3, insulin, and

11 urinary estrogen and progesterone for each subject were utilized in statistical analyses. All data

12 are presented as the mean \pm SEM.

\section{3. RESULTS}

\section{$15 \quad 3.1$ Participant Characteristics}

16 Participant characteristics are summarized in Table 1. Groups did not differ $(p>0.05)$ in

17 age, height, weight and BMI. All subjects were weight stable throughout the study period. Age

18 of menarche was similar ( $p>0.05$ ) among all groups, but gynecologic age (chronologic age minus

19 age of menarche; years) was lowest ( $p=0.024$; main effect) in ExAmen ( $9.5 \pm 1.4)$, compared

20 with ExOv (12.6 \pm 0.9) and SedOv (15.6 \pm 1.8$)$ women. Average cycle length was $28.5 \pm 0.8$ and

$2128.9 \pm 0.7$ days in the SedOv and ExOv groups, respectively. Average duration of amenorrhea for

22 the ExAmen group was $247 \pm 48$ days. Exercising groups had higher $(p<0.001$; main effect)

23 cardiorespiratory fitness and lower ( $p<0.05$; main effect) percent body fat, and total and central

24 fat mass (kg) compared with sedentary women. ExAmen trended toward lower $(p=0.054$; main

25 effect) peripheral fat mass compared with SedOv. REE adjusted for FFM was lower $(p=0.002$; 
1 main effect) in ExAmen compared with all other groups. Total BMD, L1-L4 BMD and L1-L4 Z-

2 score were lower $(p<0.05$; main effect) in ExAmen compared with ExOv.

$4 \quad 3.2$ Serum Measures

$5 \quad$ Serum measures are shown in Table 2. Log adiponectin trended toward higher

6 concentrations ( $p=0.056$; main effect) in ExAmen compared with SedOv and ExOv women.

7 When log adiponectin was adjusted for fat mass (kg), ExAmen had significantly higher ( $p=0.001$;

8 main effect) concentrations compared with all other groups (Figure 1). As previously reported by

9 our group [6, 20], serum leptin concentrations were lower ( $p=0.012$; main effect) in exercising

10 women (ExOv, $4.7 \pm 0.7 \mathrm{ng} / \mathrm{ml}$; ExAmen, $4.5 \pm 1.0 \mathrm{ng} / \mathrm{ml}$; log adjusted values) compared to

11 sedentary women $(9.3 \pm 1.8 \mathrm{ng} / \mathrm{ml})$. Consistent with our previous data [25], serum ghrelin

12 concentrations $(\mathrm{pg} / \mathrm{ml})$ were higher $(p=0.011$; main effect $)$ in ExAmen $(1939.7 \pm 215.9)$

13 compared with SedOv (1385.2 \pm 99.7) and ExOv (1397 \pm 87.3), and serum TT3 (ng/dl) lower in

14 ExAmen (89.1 \pm 8.8), compared with SedOv (111.5 \pm 2.7$)$ and ExOv (103.3 \pm 3.1). Serum

15 insulin concentrations were similar ( $4.7 \pm 0.3 \mu \mathrm{IU} / \mathrm{ml}$; pooled value; $p=0.327$; main effect $)$ among 16 the groups.

\section{$18 \quad 3.3$ Urinary Measures}

19 As previously reported by our group [6, 7] urinary E1G and log PdG exposure (see Table

20 2), determined by the AUC trapezoidal method, was significantly lower ( $p=0.002$ and $p=0.001$ for

21 E1G and log PdG, respectively; main effects) in the ExAmen group compared with ExOv and

22 SedOv groups across the menstrual cycle/monitoring period (Figure 2). E1G UC remained

23 significantly lower in ExAmen after adjusting for fat mass. LH levels, assessed in ovulatory

24 women only, were similar $(p>0.05)$ between SedOv and ExOv groups. 


\section{$1 \quad 3.4$ Adiponectin Correlates}

Using pooled data (Table 3), we report that regional fat mass (i.e., trunk and peripheral)

3 and total fat mass, percent body fat, log adjusted leptin, ghrelin, TT3, REE adjusted for FFM,

4 insulin and urinary E1G AUC measures are not correlated with log adiponectin in adult

5 physically active and inactive women. Log adiponectin adjusted for fat mass was also not

6 associated with ghrelin, REE adjusted for FFM, TT3, insulin, and urinary E1G AUC. In contrast,

7 both log adiponectin and log adiponectin adjusted for fat mass were positively associated with

8 age at menarche ( $\mathrm{r}=0.461, p=0.006 ; \mathrm{r}=0.352, p=0.048$; respectively), and negatively associated

9 with gynecologic age ( $\mathrm{r}=-0.379, p=0.027 ; \mathrm{r}=-0.489, p=0.005$; respectively) and all bone

10 measures (see Table 3). As expected, log leptin was positively associated $(p<0.05)$ with all

11 indices of adiposity. In contrast, log adiponectin adjusted for fat mass (kg) was negatively

12 associated $(p<0.05)$ with all indices of adiposity.

13 Examining each group independently, (i.e., SedOv, ExOv, and ExAmen), serum log

14 adiponectin was not associated ( $p>0.05)$ with urinary E1G AUC, age, age at menarche,

15 gynecologic age, insulin, ghrelin, TT3, REE adjusted for FFM, log leptin or measures of body fat

16 in any group. In ExOv only, log adiponectin was inversely associated $(p<0.05)$ with all bone

17 measures, except total BMD ( $p=0.130)$. For log adiponectin adjusted for fat mass, SedOv group

18 only demonstrated negative associations with most measures of fat mass, including percent body

19 fat $(\mathrm{r}=-0.730, p=0.026)$, central fat mass $(\mathrm{r}=-0.755, p=0.019)$, total fat mass $(\mathrm{r}=-0.741$,

$20 p=0.022)$, and BMI $(\mathrm{r}=-0.799, p=0.010)$. In ExAmen only, urinary E1G AUC trended toward a

21 positive association with log adiponectin adjusted for fat mass ( $\mathrm{r}=0.657, p=0.077$.

\section{$23 \quad 3.5$ Predictors of Adiponectin}

24 Using pooled data for stepwise linear regression, we entered variables that have been

25 previously reported to be associated with adiponectin, including leptin, ghrelin, fat mass, BMI,

26 and nutritional status [8, 14, 17, 26]. Specifically, variables in the regression model included: 
1 BMI, log leptin, ghrelin, TT3, and central and peripheral fat mass. Both fat mass measures were

2 included in the model to determine whether site specific body fat deposition predicted

3 adiponectin levels. No predictors of log adiponectin were identified. In contrast, fat mass

4 adjusted log adiponectin was predicted solely by trunkal fat mass (see Table 4), which explained

$5 \sim 41 \%$ of the variance (adjusted $R^{2}=0.413, p<0.001$ ). Variables included in this model were the

6 same as that for log adiponectin.

\section{$8 \quad 3.6$ Associations with, and Predictors of, Gonadal Status}

9 Using pooled data for bivariate analyses, nutritionally mediated metabolic indicators of 10 energy deficiency, such as TT3 and REE adjusted for FFM, were independently and positively

11 associated with E1G AUC in the current study $(\mathrm{r}=0.463, p=0.007 ; \mathrm{r}=0.389, p=0.030$,

12 respectively). In contrast, log leptin, ghrelin, and regional and total fat mass were not associated

13 with E1G AUC. Urinary log PdG AUC demonstrated positive associations with E1G AUC

$14(\mathrm{r}=0.575, p=0.001)$ and TT3 $(\mathrm{r}=0.419, p=0.014)$. LH levels, assessed in ovulatory women only,

15 did not correlate with any hormonal, nutritional, body compositional or bone health measures

16 when analyzed using SedOv and ExOv women only.

17 Due to detection of fewer significant correlates for PdG AUC or LH compared with E1G

18 AUC, linear regression models for predictors of gondal status focused on E1G AUC. Using

19 pooled data for stepwise linear regression, we entered variables of interest, namely adiponectin, in

20 addition to factors that have previously been reported to be associated with circulating estrogen

21 levels, including total fat mass, TT3, leptin, and ghrelin [8, 14, 20]. For the first time, we report

22 herein that in adult female athletes with secondary amenorrhea log adiponectin concentrations,

23 both adjusted and unadjusted for fat mass, do not predict $(p>0.05)$ estrogen exposure, and as such,

24 fail to predict gonadal status. Similarly, other nutritionally regulated hormones, such as log leptin

25 and ghrelin, and total fat mass, do not predict gonadal status. The strongest, and only, predictor

26 of gonadal status was identified as TT3 (adjusted $R^{2}=0.228, p=0.004$; see Table 4). 


\section{$2 \quad 3.7$ Associations with, and Predictors of, Bone Health}

3 Using pooled data, the current study shows that both lumbar and total BMD were

4 significantly $(p<0.05)$ negatively correlated with log adiponectin (Table 3; Figure 3). Urinary

5 E1G AUC trended ( $p=0.074)$ toward a significant positive association with L1-L4 BMD, but was

6 not associated with total BMD. Using pooled data for stepwise linear regression, we entered log

7 adiponectin, log leptin, ghrelin, insulin, E1G AUC, TT3 and BMI into two separate prediction

8 models, one for L1-L4 BMD and one for total BMD. All of the chosen variables have been

9 previously shown to be associated with bone health in humans [27-31]. Log adiponectin and E1G

10 AUC collectively contributed approximately 37\% to the variability of L1-L4 BMD (adjusted

$11 R^{2}=0.374, p=0.045$; see Table 4). Total BMD was negatively predicted by log adiponectin

12 (adjusted $R^{2}=0.188, p=0.009$; see Table 4). Although not determined to be an outlier, removal of

13 the data point showing very high BMD and very low adiponectin (see Figure 3) did not

14 significantly alter the predictors of bone health or the line of best fit between log adiponectin and

\section{L1-L4 BMD.}

16 To account for the potential effects of exercise training on BMD [32], we examined bone

17 health associations in exercising women only (ExOv and ExAmen). Both log adiponectin and log

18 adiponectin adjusted for fat mass were significantly and negatively associated L1-L4 BMD (r= -

19 0.612, $p=0.002 ; \mathrm{r}=-0.561, p=0.005$, respectively $)$ and total $\operatorname{BMD}(\mathrm{r}=-0.542, p=0.008 ; \mathrm{r}=-0.499$,

$20 p=0.015$; respectively). E1G AUC trended toward a positive association with L1-L4 BMD ( $\mathrm{r}=$

$210.417 ; p=0.054)$. Predictors of bone health in exercising women only were also carried out. For

22 both L1-L4 BMD and total BMD, each model was positively predicted by log adiponectin and

23 E1G AUC (adjusted $R^{2}=0.467, F(1,19)=10.20, p=0.001$; adjusted $R^{2}=0.333, F(1,29)=6.232$,

$24 p=0.008$; respectively). Variables included in these models were the same as that used for the

25 pooled data set. 


\section{4. DISCUSSION}

2 The novel findings of this study are: 1) in comparison with adult eumenorrheic ovulatory

3 sedentary and exercising women, physically active adult women with EDAA demonstrate

4 elevated adiponectin concentrations relative to their fat mass; 2) log adiponectin adjusted for fat

5 mass was predicted by trunkal fat mass; 3) lumbar bone health was predicted by log adiponectin

6 and E1G AUC, while total body BMD was predicted solely by log adiponectin; and 4) the

7 strongest predictor of gonadal status did not include secretory products of adipose tissue, namely

8 adiponectin and leptin, but rather, nutritionally mediated metabolic hormones, specifically, TT3.

9 While these data are associative in nature and do not relate to causality, these findings suggest

10 that in physically active women with EDAA: 1) elevated adiponectin and hypoestrogenemia are

11 independently, and in combination, associated with impaired bone health; 2) circulating

12 adiponectin is not related to nutritionally or hormonally regulated factors or gonadal status, but is

13 inversely associated with trunkal fat mass; and 3) nutritionally mediated metabolic hormones,

14 specifically TT3, likely play a role in mediating gonadal status via energy deficiency related

15 mechanisms. The clinical relevance of these findings is not yet known.

174.1 Adiponectin in Amenorrheic Athletes

18 In the current study, we report for the first time that ExAmen demonstrate elevated log

19 adiponectin concentrations relative to their fat mass when compared with ExOv and SedOv

20 women, supporting an inverse relationship between body fat and adiponectin concentrations. This

21 finding is consistent with previous studies demonstrating an inverse relationship between serum

22 adiponectin levels and parameters of overall adiposity, such as fat mass, BMI and percent fat

23 mass in humans [12, 13]. It is also in agreement with data showing significantly elevated

24 adiponectin adjusted for fat mass in female anorexia nervosa patients compared with healthy age-

25 matched adolescents [14]. In contrast, however, we report that log adiponectin concentrations that

26 were not adjusted for fat mass were both similar between the groups and not related to whole or 
1 regional fat mass. While this observation is not in keeping with the reported inverse association

2 between fat mass and adiponectin [12, 13], others have similarly reported both comparable

3 adiponectin levels and no association between total fat mass and serum adiponectin in female

4 adolescent athletes presenting with secondary and primary amenorrhea compared with their

5 eumenorrheic counterpart [9]. No associations of adiponectin with total fat mass have also been

6 reported in hypoestrogenic adolescent anorexia nervosa patients [14]. Reasons for equivocal

7 findings in the current study, and the above studies [9, 12-14] are unclear, but may be related, in

8 part, to a number of factors, including adjustment of adiponectin concentrations relative to fat

9 mass, differences in the adiponectin assay used (i.e., high molecular weight versus total

10 adiponectin), the chosen population, and gonadal status.

11

\subsection{Adiponectin and Bone}

13 Increases in BMD above levels seen in age-matched sedentary females are notably

14 expressed in females between 20-25 years of age participating in load bearing exercise training

15 [32]. In the present study, however, we report that despite participation in regular load-bearing

16 exercise training, ExAmen women demonstrate lower lumbar and total BMD in association with

17 elevated adiponectin levels. Impaired bone health in weight-stable amenorrheic athletes has

18 previously been shown to be associated with hypogonadism, particularly in the presence of an

19 energy deficiency [30]. Consistent with this finding, we report here that E1G AUC was a

20 significant contributor to our prediction model of L1-L4 BMD, and that ExAmen demonstrate

21 significantly lower TT3 concentrations compared with SedOv and ExOv. Low TT3 levels are

22 recognized as a marker of under-nutrition and energy deficiency [6], indicating that the

23 hypoestrogenic physically active women in the present study were likely energy deficient.

24 Despite these alterations, we failed to demonstrate associations between bone health and TT3.

25 Other factors known to impact bone health were also not associated with lumbar or total BMD,

26 including ghrelin, leptin, and insulin. This is of interest since ghrelin and insulin are known to 
1 have stimulatory effects on osteoblastic activity [27, 28], and leptin is known to modulate bone

2 turnover through complex central and peripheral effects [29]. In contrast, however, we report

3 here, for the first time, that spine and total BMD are inversely associated with circulating log

4 adiponectin concentrations in adult amenorrheic physically active women. Similarly, other

5 studies report decreased BMD in association with increased circulating adiponectin levels in

6 adolescent hypoestrogenic amenorrheic anorexia nervosa patients [14], and adolescent

7 amenorrheic female athletes [9]. These findings, and that of the current study, are in keeping with

8 the documented stimulatory affect of adiponectin on osteoclastic activity in humans [33], but are

9 in contrast to the reported increased osteoblastic and decreased osteoclastic activity in animal

10 models [34]. Collectively, our findings and those of others [14, 27, 29, 33, 34] underscore the

11 complexity of the interactions between nutritionally and metabolically regulated hormones in

12 determining bone metabolism.

13

\section{$14 \quad 4.3$ Adiponectin and Gonadal Status}

15 Among our study groups, physically active women with EDAA demonstrated the lowest

16 estrogen exposure and the highest adiponectin concentrations compared with ovulatory groups,

17 suggesting a possible inhibitory role of estrogen on circulating adiponectin. However, we failed

18 to identify an association between urinary estrogen exposure over time and serum log adiponectin

19 concentrations. In agreement with this, others similarly document no correlation between serum

20 adiponectin and estrogen levels among premenopausal and postmenopausal women [35], and

21 among amenorrheic and eumenorrheic adolescent athletes [9]. Lack of association between

22 estrogen and adiponectin concentrations in the current study suggests that estrogen deficiency in

23 amenorrheic athletes is likely reflective of menstrual status per se and that other factors

24 coincident with amenorrhea impact serum adiponectin levels in our chosen population. Such

25 factors could include any combination of the well documented hormonal and metabolic

26 alterations previously reported in amenorrheic athletes, including hypoglycemia [5], 
1 hypoinsulinemia [5, 36], hypercortisolemia [5], hypothyroidemia [36], reduced REE [37],

2 decreased ratio in measured versus predicted REE [10], lower leptin levels [36], and elevated

3 ghrelin [25] and peptide YY [10] levels. While we observed that TT3 and REE adjusted for FFM

4 were independently associated with gonadal status, we did not detect any relationship with

5 adiponectin, ghrelin, insulin, or leptin. This finding suggests that metabolic rather than

6 nutritionally regulated factors may mediate gonadal status in adult amenorrheic physically active

7 premenopausal women.

8

$9 \quad 4.4$ Adiponectin and Body Fat Distribution

10 In keeping with the well documented effect of regular physical activity on body

11 composition, we observed greater central and peripheral fat mass in sedentary women compared

12 with exercising women. Specifically, ExAmen women demonstrated significantly lower regional

13 and total fat mass compared with SedOv, and non-significantly lower regional and total fat mass

14 compared with ExOv women. Similar body composition in amenorrheic athletes has been

15 previously reported [6, 20, 25]. Using pooled data, trunkal fat mass, but not peripheral fat mass

16 or whole body fat mass, was inversely predicted by log adiponectin adjusted for fat mass. This

17 finding is consistent with recent in vitro evidence demonstrating that the rate of adiponectin

18 secretion is approximately threefold higher from visceral compared with subcutaneous fat [16].

19 Similarly, others have also reported an inverse association between serum adiponectin and

20 visceral fat [38]. These findings [16, 38], and that of the current study, supports the postulate that

21 distribution of body fat rather than total fat amount may be important to adiponectin metabolism.

22 This hypothesis awaits further investigation.

\section{$24 \quad 4.5$ Limitations}

25 While the primary objectives of this study were to examine serum adiponectin levels in

26 physically active women with EDAA and to examine whether this nutritionally regulated 
1 hormone could predict gonadal status or bone health, the current study only examines

2 associations, not cause and effect. In addition, our chosen method to determine predictors of

3 adiponectin, gonadal status and bone health may not be optimal. As such, these associations and

4 predictions should be interpreted appropriately. We also failed to examine differences between

5 varying molecular weight adiponectin molecules, and we did not examine all possible urinary

6 estrone conjugates. It is possible that differences exist between low, medium, high, and total

7 molecular weight adiponectin, and between the different estrone conjugates in adult amenorrheic

8 athletes. Finally, our study groups were of small sample size, and may have therefore affected

9 our ability to detect true associations where present. As such, our findings should be interpreted

10 prudently.

11

\section{5. CONCLUSION}

13 We demonstrate for the first time that total serum adiponectin levels relative to fat mass

14 are elevated in exercising adult premenopausal women with EDAA. While this elevation does not

15 predict gonadal status, as determined by estrogen exposure across time, increased adiponectin

16 concentrations and estrogen exposure were independently, and in combination, associated with

17 impaired bone health. Adiponectin relative to fat mass was also inversely associated with trunkal

18 fat mass, implying that body fat distribution rather than total body fat mass may be important in

19 determining adiponectin concentrations. Markers of nutritionally mediated metabolic status,

20 namely TT3, predicted estrogen exposure, suggesting that metabolic factors likely mediate

21 gonadal status via energy deficiency related mechanisms. Although mechanisms and

22 consequences of elevated adiponectin concentrations in amenorrheic athletes remain to be

23 elucidated, the results of this study further characterize the unique endocrine profile of these

24 women. 
1 Acknowledgements: We are very grateful to the women who participated in this study.

2

3 Declaration of interest: The authors have no competing interests.

4

5 Funding: This work was supported by the Arthur Thornton Cardiopulmonary Fund, New Britain

6 General Hospital, Connecticut. 


\section{References}

[1] Loucks AB, Thuma JR. Luteinizing hormone pulsatility is disrupted at a threshold of energy availability in regularly menstruating women. J Clin Endocrinol Metab 2003;88: 297-311.

[2] Williams NI, Caston-Balderrama AL, Helmreich DL, Parfitt DB, Nosbisch C, Cameron $\mathrm{JL}$. Longitudinal changes in reproductive hormones and menstrual cyclicity in cynomolgus monkeys during strenuous exercise training: abrupt transition to exercise-induced amenorrhea. Endocrinology 2001;142: 2381-9.

[3] De Souza MJ, Toombs RJ, Scheid JL, O'Donnell E, West SL, Williams NI. High prevalence of subtle and severe menstrual disturbances in exercising women: confirmation using daily hormone measures. Hum Reprod 2009.

[4] Otis CL, Drinkwater B, Johnson M, Loucks A, Wilmore J. American College of Sports Medicine position stand. The Female Athlete Triad. Med Sci Sports Exerc 1997;29: i-ix.

[5] Laughlin GA, Yen SS. Nutritional and endocrine-metabolic aberrations in amenorrheic athletes. J Clin Endocrinol Metab 1996;81: 4301-9.

[6] O'Donnell E, Harvey PJ, Goodman JM, De Souza MJ. Long-term estrogen deficiency lowers regional blood flow, resting systolic blood pressure, and heart rate in exercising premenopausal women. Am J Physiol Endocrinol Metab 2007;292: E1401-9.

[7] De Souza MJ, Lee DK, VanHeest JL, Scheid JL, West SL, Williams NI. Severity of energy-related menstrual disturbances increases in proportion to indices of energy conservation in exercising women. Fertil Steril 2007;88: 971-5.

[8] Christo K, Cord J, Mendes N, Miller KK, Goldstein MA, Klibanski A, Misra M. Acylated ghrelin and leptin in adolescent athletes with amenorrhea, eumenorrheic athletes and controls: a cross-sectional study. Clin Endocrinol (Oxf) 2008;69: 628-33.

[9] Russell M, Stark J, Nayak S, Miller KK, Herzog DB, Klibanski A, Misra M. Peptide YY in adolescent athletes with amenorrhea, eumenorrheic athletes and non-athletic controls. Bone 2009;45: 104-9.

[10] Scheid JL, Williams NI, West SL, VanHeest JL, De Souza MJ. Elevated PYY is associated with energy deficiency and indices of subclinical disordered eating in exercising women with hypothalamic amenorrhea. Appetite 2009;52: 184-92.

[11] Rajala MW, Scherer PE. Minireview: The adipocyte--at the crossroads of energy homeostasis, inflammation, and atherosclerosis. Endocrinology 2003;144: 3765-73.

[12] Cambuli VM, Musiu MC, Incani M, Paderi M, Serpe R, Marras V, Cossu E, Cavallo MG, Mariotti S, Loche S, Baroni MG. Assessment of adiponectin and leptin as biomarkers of positive metabolic outcomes after lifestyle intervention in overweight and obese children. J Clin Endocrinol Metab 2008;93: 3051-7.

[13] Arita Y, Kihara S, Ouchi N, Takahashi M, Maeda K, Miyagawa J, Hotta K, Shimomura I, Nakamura T, Miyaoka K, Kuriyama H, Nishida M, Yamashita S, Okubo K, Matsubara K, 
1 Muraguchi M, Ohmoto Y, Funahashi T, Matsuzawa Y. Paradoxical decrease of an adipose-

2 specific protein, adiponectin, in obesity. Biochem Biophys Res Commun 1999;257: 79-83.

[14] Misra M, Miller KK, Cord J, Prabhakaran R, Herzog DB, Goldstein M, Katzman DK, Klibanski A. Relationships between serum adipokines, insulin levels, and bone density in girls with anorexia nervosa. J Clin Endocrinol Metab 2007;92: 2046-52.

[15] Tagami T, Satoh N, Usui T, Yamada K, Shimatsu A, Kuzuya H. Adiponectin in anorexia nervosa and bulimia nervosa. J Clin Endocrinol Metab 2004;89: 1833-7.

[16] Perrini S, Laviola L, Cignarelli A, Melchiorre M, De Stefano F, Caccioppoli C, Natalicchio A, Orlando MR, Garruti G, De Fazio M, Catalano G, Memeo V, Giorgino R, Giorgino F. Fat depot-related differences in gene expression, adiponectin secretion, and insulin action and signalling in human adipocytes differentiated in vitro from precursor stromal cells. Diabetologia 2008;51: 155-64.

[17] Pannacciulli N, Bunt JC, Ortega E, Funahashi T, Salbe AD, Bogardus C, Krakoff J. Lower total fasting plasma adiponectin concentrations are associated with higher metabolic rates. J Clin Endocrinol Metab 2006;91: 1600-3.

[18] Cnop M, Havel PJ, Utzschneider KM, Carr DB, Sinha MK, Boyko EJ, Retzlaff BM, Knopp RH, Brunzell JD, Kahn SE. Relationship of adiponectin to body fat distribution, insulin sensitivity and plasma lipoproteins: evidence for independent roles of age and sex. Diabetologia 2003;46: 459-69.

[19] Chalvatzas N, Dafopoulos K, Kosmas G, Kallitsaris A, Pournaras S, Messinis IE. Effect of ovarian hormones on serum adiponectin and resistin concentrations. Fertil Steril 2008.

[20] O'Donnell E, Harvey PJ, De Souza MJ. Relationships between vascular resistance and energy deficiency, nutritional status and oxidative stress in oestrogen deficient physically active women. Clin Endocrinol (Oxf) 2009;70: 294-302.

[21] De Souza MJ, Miller BE, Loucks AB, Luciano AA, Pescatello LS, Campbell CG, Lasley BL. High frequency of luteal phase deficiency and anovulation in recreational women runners: blunted elevation in follicle-stimulating hormone observed during luteal-follicular transition. $\mathrm{J}$ Clin Endocrinol Metab 1998;83: 4220-32.

[22] Saltin B, Astrand PO. Maximal oxygen uptake in athletes. J Appl Physiol 1967;23: 3538.

[23] De Souza MJ, Williams NI. Physiological aspects and clinical sequelae of energy deficiency and hypoestrogenism in exercising women. Hum Reprod Update 2004;10: 433-48.

[24] Weir JB. New methods for calculating metabolic rate with special reference to protein metabolism. J Physiol 1949;109: 1-9.

[25] De Souza MJ, Leidy HJ, O'Donnell E, Lasley B, Williams NI. Fasting ghrelin levels in physically active women: relationship with menstrual disturbances and metabolic hormones. $\mathrm{J}$ Clin Endocrinol Metab 2004;89: 3536-42. 
1 [26] Bobbert T, Rochlitz H, Wegewitz U, Akpulat S, Mai K, Weickert MO, Mohlig M, 2 Pfeiffer AF, Spranger J. Changes of adiponectin oligomer composition by moderate weight 3 reduction. Diabetes 2005;54: 2712-9.

4

[27] Kim SW, Her SJ, Park SJ, Kim D, Park KS, Lee HK, Han BH, Kim MS, Shin CS, Kim SY. Ghrelin stimulates proliferation and differentiation and inhibits apoptosis in osteoblastic MC3T3-E1 cells. Bone 2005;37: 359-69.

[28] Thrailkill KM, Lumpkin CK, Jr., Bunn RC, Kemp SF, Fowlkes JL. Is insulin an anabolic agent in bone? Dissecting the diabetic bone for clues. Am J Physiol Endocrinol Metab 2005;289: E735-45.

[29] Lee NJ, Wong IP, Baldock PA, Herzog H. Leptin as an endocrine signal in bone. Curr Osteoporos Rep 2008;6: 62-6.

[30] De Souza MJ, West SL, Jamal SA, Hawker GA, Gundberg CM, Williams NI. The presence of both an energy deficiency and estrogen deficiency exacerbate alterations of bone metabolism in exercising women. Bone 2008;43: 140-8.

[31] Kaufman BA, Warren MP, Dominguez JE, Wang J, Heymsfield SB, Pierson RN. Bone density and amenorrhea in ballet dancers are related to a decreased resting metabolic rate and lower leptin levels. J Clin Endocrinol Metab 2002;87: 2777-83.

[32] Duncan CS, Blimkie CJ, Cowell CT, Burke ST, Briody JN, Howman-Giles R. Bone mineral density in adolescent female athletes: relationship to exercise type and muscle strength. Med Sci Sports Exerc 2002;34: 286-94.

[33] Luo XH, Guo LJ, Xie H, Yuan LQ, Wu XP, Zhou HD, Liao EY. Adiponectin stimulates RANKL and inhibits OPG expression in human osteoblasts through the MAPK signaling pathway. J Bone Miner Res 2006;21: 1648-56.

[34] Shinoda Y, Yamaguchi M, Ogata N, Akune T, Kubota N, Yamauchi T, Terauchi Y, Kadowaki T, Takeuchi Y, Fukumoto S, Ikeda T, Hoshi K, Chung UI, Nakamura K, Kawaguchi $\mathrm{H}$. Regulation of bone formation by adiponectin through autocrine/paracrine and endocrine pathways. J Cell Biochem 2006;99: 196-208.

[35] Sieminska L, Wojciechowska C, Niedziolka D, Marek B, Kos-Kudla B, Kajdaniuk D, Nowak M. Effect of postmenopause and hormone replacement therapy on serum adiponectin levels. Metabolism 2005;54: 1610-4.

[36] Thong FS, McLean C, Graham TE. Plasma leptin in female athletes: relationship with body fat, reproductive, nutritional, and endocrine factors. J Appl Physiol 2000;88: 2037-44.

[37] Myburgh KH, Berman C, Novick I, Noakes T, Lambert E. Decreased resting metabolic rate in ballet dancers with menstrual irregularity. Int J Sport Nutr 1999;9: 285-94.

[38] Lenchik L, Register TC, Hsu FC, Lohman K, Nicklas BJ, Freedman BI, Langefeld CD, Carr JJ, Bowden DW. Adiponectin as a novel determinant of bone mineral density and visceral fat. Bone 2003;33: 646-51. 
1 Figure 1. Bar chart showing serum adiponectin levels adjusted for fat mass (mg/L/kg) among the 2 study groups. * $p=0.001$ (main effect) ExAmen vs SedOv and ExOv.

3

$4 \quad$ Figure 2. Bar chart showing estrogen exposure across the menstrual cycle, or 30-day monitoring 5 period, by means of daily urinary E1G area under the curve analysis for each study group.

$6 * p=0.002$ (main effect) ExAmen vs SedOv and ExOv.

7

8 Figure 3. Scatterplot showing the relationship between lumbar spine BMD $\left(\mathrm{g} / \mathrm{cm}^{2}\right)$ and log

9 adiponectin concentrations ( $\mathrm{ng} / \mathrm{mL})$ for all groups $\left(\mathrm{r}=-0.538 ; p=0.001 ; R^{2}=0.289\right)$.

10 
Table 1. Subject characteristics of the study groups.

\begin{tabular}{|c|c|c|c|c|}
\hline & $\begin{array}{l}\text { SedOv } \\
(n=10)\end{array}$ & $\begin{array}{l}\mathrm{ExOv} \\
(n=15)\end{array}$ & $\begin{array}{c}\text { ExAmen } \\
(n=9)\end{array}$ & $\begin{array}{l}P \text { (main } \\
\text { effect) }\end{array}$ \\
\hline \multicolumn{5}{|l|}{ Demographics } \\
\hline Age (years) & $27.8 \pm 1.8$ & $24.7 \pm 0.9$ & $24.0 \pm 1.5$ & 0.146 \\
\hline Age of menarche (years) & $12.5 \pm 0.4$ & $12.2 \pm 0.3$ & $13.3 \pm 0.6$ & 0.177 \\
\hline Gynecologic age (years) & $15.6 \pm 1.8$ & $12.6 \pm 0.9$ & $9.5 \pm 1.4^{\mathrm{a}}$ & 0.024 \\
\hline Weight (kg) & $58.2 \pm 2.4$ & $57.5 \pm 1.3$ & $57.5 \pm 2.0$ & 0.952 \\
\hline Height (cm) & $161.6 \pm 1.6$ & $164.8 \pm 1.3$ & $165.3 \pm 1.3$ & 0.181 \\
\hline BMI $\left(\mathrm{kg} / \mathrm{m}^{2}\right)$ & $22.4 \pm 0.8$ & $21.3 \pm 0.4$ & $21.1 \pm 0.7$ & 0.279 \\
\hline Body fat total (\%) & $31.0 \pm 2.2^{b}$ & $24.3 \pm 1.3$ & $19.9 \pm 2.2$ & 0.002 \\
\hline Body FM total (kg) & $17.6 \pm 1.9^{\mathrm{b}}$ & $13.3 \pm 0.9$ & $11.2 \pm 1.5$ & 0.014 \\
\hline Trunk FM (kg) & $8.53 \pm 1.03^{\mathrm{b}}$ & $6.2 \pm 0.6$ & $4.9 \pm 0.7$ & 0.010 \\
\hline Peripheral FM (kg) & $8.4 \pm 0.9$ & $6.6 \pm 0.4$ & $5.8 \pm 0.8$ & 0.054 \\
\hline $\mathrm{VO}_{2} \max (\mathrm{ml} / \mathrm{kg} / \mathrm{min})$ & $38.6 \pm 1.3^{\mathrm{b}}$ & $46.3 \pm 1.3$ & $45.4 \pm 1.2$ & 0.001 \\
\hline REE/FFM (kcal/day/kg) & $33.4 \pm 0.9$ & $31.9 \pm 0.7$ & $28.7 \pm 0.9^{\mathrm{a}}$ & 0.002 \\
\hline L1-L4 BMD (g/cm²) & $1.18 \pm 0.04$ & $1.28 \pm 0.05$ & $1.11 \pm 0.04^{\mathrm{C}}$ & 0.026 \\
\hline L1-L4 BMD Z-score & $0.19 \pm 0.30$ & $1.05 \pm 0.40$ & $-0.48 \pm 0.2^{\mathrm{C}}$ & 0.015 \\
\hline Total BMD (g/cm²) & $1.14 \pm 0.02$ & $1.20 \pm 0.02$ & $1.13 \pm 0.02^{\mathrm{C}}$ & 0.028 \\
\hline Total BMD Z-score & $0.47 \pm 0.22$ & $1.24 \pm 0.22^{\mathrm{d}}$ & $0.34 \pm 0.27$ & 0.019 \\
\hline
\end{tabular}

Values are mean \pm SEM. BMI, body mass index; $\mathrm{VO}_{2}$ max, maximal oxygen uptake; EIG, urinary estrone 3-glucuronide; AUC, area under the curve; FM, fat mass; REE, resting energy expenditure; FFM, fat free mass; BMD, bone mineral density.

${ }^{\mathrm{a}}$ ExAmen vs. SedOv \& ExOv

${ }^{\mathrm{b}}$ SedOv vs. ExOv \& ExAmen

${ }^{\mathrm{c}}$ ExAmen vs. ExOv

${ }^{\mathrm{d}}$ ExOv vs. ExAmen and SedOv 
Table 2. Reproductive hormones and serum measures for the study groups.

\begin{tabular}{lcccc}
\hline & $\begin{array}{c}\text { SedOv } \\
(\mathrm{n}=10)\end{array}$ & $\begin{array}{c}\text { ExOv } \\
(\mathrm{n}=15)\end{array}$ & $\begin{array}{c}\text { ExAmen } \\
(\mathrm{n}=9)\end{array}$ & $\begin{array}{c}P \text { (main } \\
\text { effect) }\end{array}$ \\
\hline Reproductive Hormones & & & & \\
E1G AUC (ng/ml) & $1864.9 \pm 232.7$ & $2047.9 \pm 256.4$ & $648.9 \pm 160.3^{\mathrm{a}}$ & 0.002 \\
E1G AUC/FM (ng/ml/kg) & $126.6 \pm 25.4$ & $173.3 \pm 23.9$ & $69.9 \pm 17.2^{\mathrm{b}}$ & 0.019 \\
& & & & \\
Serum Measures & & & & \\
Adiponectin (mg/L) & $8.2 \pm 0.8$ & $9.4 \pm 1.3$ & $14.4 \pm 2.7^{\mathrm{c}}$ & 0.028 \\
Adiponectin/FM (mg/L/kg) & $0.5 \pm 0.1$ & $0.7 \pm 0.1$ & $1.4 \pm 0.2^{\mathrm{a}}$ & $<0.001$ \\
Log Adiponectin (mg/L) & $3.8 \pm 0.1$ & $3.9 \pm 0.1$ & $4.1 \pm 0.1^{\mathrm{a}}$ & 0.056 \\
Log Adiponectin/FM (mg/L/kg) & $2.7 \pm 0.1$ & $2.8 \pm 0.1$ & $3.1 \pm 0.1^{\mathrm{a}}$ & 0.001 \\
\hline
\end{tabular}

Values are mean \pm SEM. BMI, body mass index; $\mathrm{VO}_{2}$ max, maximal oxygen uptake; EIG, urinary estrone 3-glucuronide; AUC, area under the curve; FM, fat mass.

${ }^{a}$ ExAmen vs. SedOv \& ExOv

${ }^{\mathrm{b}}$ ExAmen vs. ExOv

${ }^{\mathrm{c}}$ ExAmen vs. SedOv 
Table 3: Bivariate correlates between hormones, body composition and bone health.*

\begin{tabular}{|c|c|c|c|c|c|c|c|c|c|c|c|}
\hline & $\begin{array}{l}\text { E1G } \\
\text { AUC }\end{array}$ & $\begin{array}{c}\text { Log } \\
\text { Leptin }\end{array}$ & TT3 & Ghrelin & Insulin & $\begin{array}{c}\text { Total } \\
\text { FM }\end{array}$ & $\begin{array}{c}\text { Trunk } \\
\text { FM }\end{array}$ & $\begin{array}{l}\text { Periph. } \\
\text { FM }\end{array}$ & $\begin{array}{l}\text { REE/ } \\
\text { FFM }\end{array}$ & $\begin{array}{l}\text { L1-L4 } \\
\text { BMD }\end{array}$ & $\begin{array}{l}\text { Total } \\
\text { BMD }\end{array}$ \\
\hline $\begin{array}{l}\text { Log Adipon. } \\
\text { (mg/L) }\end{array}$ & $\begin{array}{l}\mathrm{r}=-0.014 \\
\mathrm{p}=0.940\end{array}$ & $\begin{array}{c}r=0.162 \\
p=0.353\end{array}$ & $\begin{array}{l}\mathrm{r}=0.200 \\
\mathrm{p}=0.249\end{array}$ & $\begin{array}{l}r=0.030 \\
p=0.865\end{array}$ & $\begin{array}{l}r=-0.239 \\
p=0.166\end{array}$ & $\begin{array}{l}r=0.003 \\
p=0.987\end{array}$ & $\begin{array}{l}\mathrm{r}=0.098 \\
\mathrm{p}=0.589\end{array}$ & $\begin{array}{l}r=0.114 \\
p=0.529\end{array}$ & $\begin{array}{l}r=-0.106 \\
p=0.558\end{array}$ & $\begin{array}{l}r=-0.538 \\
p=0.001\end{array}$ & $\begin{array}{l}r=-0.440 \\
p=0.010\end{array}$ \\
\hline $\begin{array}{l}\text { E1G AUC } \\
(\mathrm{ng} / \mathrm{ml})\end{array}$ & - & $\begin{array}{l}r=0.021 \\
p=0.906\end{array}$ & $\begin{array}{l}r=0.463 \\
p=0.007\end{array}$ & $\begin{array}{l}r=0.097 \\
p=0.593\end{array}$ & $\begin{array}{l}r=-0.003 \\
p=0.985\end{array}$ & $\begin{array}{l}r=0.071 \\
p=0.705\end{array}$ & $\begin{array}{l}r=0.117 \\
p=0.531\end{array}$ & $\begin{array}{l}r=0.071 \\
p=0.705\end{array}$ & $\begin{array}{l}r=0.389 \\
p=0.030\end{array}$ & $\begin{array}{l}r=0.320 \\
p=0.074\end{array}$ & $\begin{array}{l}r=0.182 \\
p=0.328\end{array}$ \\
\hline $\begin{array}{l}\text { Log Leptin } \\
\text { (ng/ml) }\end{array}$ & & - & $\begin{array}{l}r=0.309 \\
p=0.070\end{array}$ & $\begin{array}{l}r=-0.202 \\
p=0.245\end{array}$ & $\begin{array}{l}\mathrm{r}=0.228 \\
\mathrm{p}=0.189\end{array}$ & $\begin{array}{l}r=0.743 \\
p=0.000\end{array}$ & $\begin{array}{l}r=0.681 \\
p=0.000\end{array}$ & $\begin{array}{l}r=0.731 \\
p=0.000\end{array}$ & $\begin{array}{l}r=0.479 \\
p=0.005\end{array}$ & $\begin{array}{l}r=-0.105 \\
p=0.556\end{array}$ & $\begin{array}{l}r=-0.113 \\
p=0.532\end{array}$ \\
\hline TT3 (ng/dl) & & & - & $\begin{array}{l}r=-0.242 \\
p=0.161\end{array}$ & $\begin{array}{l}r=0.277 \\
p=0.108\end{array}$ & $\begin{array}{l}r=0.350 \\
p=0.046\end{array}$ & $\begin{array}{l}\mathrm{r}=0.308 \\
\mathrm{p}=0.082\end{array}$ & $\begin{array}{l}r=0.359 \\
p=0.040\end{array}$ & $\begin{array}{l}r=0.406 \\
p=0.019\end{array}$ & $\begin{array}{l}r=0.008 \\
p=0.963\end{array}$ & $\begin{array}{l}r=0.086 \\
p=0.636\end{array}$ \\
\hline $\begin{array}{l}\text { Ghrelin } \\
\text { (pg/ml) }\end{array}$ & & & & 1 & $\begin{array}{l}r=-0.106 \\
p=0.544\end{array}$ & $\begin{array}{l}r=-0.260 \\
p=0.143\end{array}$ & $\begin{array}{l}r=-0.310 \\
p=0.079\end{array}$ & $\begin{array}{l}r=-0.173 \\
p=0.337\end{array}$ & $\begin{array}{l}r=-0.407 \\
p=0.017\end{array}$ & $\begin{array}{l}r=0.029 \\
p=0.869\end{array}$ & $\begin{array}{l}r=0.145 \\
p=0.422\end{array}$ \\
\hline $\begin{array}{l}\text { Insulin } \\
\text { (uIU/ml) }\end{array}$ & & & & & - & $\begin{array}{l}r=0.056 \\
p=0.756\end{array}$ & $\begin{array}{l}r=0.096 \\
p=0.597\end{array}$ & $\begin{array}{l}\mathrm{r}=0.001 \\
\mathrm{p}=0.997\end{array}$ & $\begin{array}{l}\mathrm{r}=0.281 \\
\mathrm{p}=0.113\end{array}$ & $\begin{array}{l}\mathrm{r}=0.247 \\
\mathrm{p}=0.158\end{array}$ & $\begin{array}{l}r=0.121 \\
p=0.502\end{array}$ \\
\hline $\begin{array}{l}\text { Total FM } \\
(\mathrm{kg})\end{array}$ & & & & & & - & $\begin{array}{l}r=0.944 \\
p=0.000\end{array}$ & $\begin{array}{l}\mathbf{r}=\mathbf{0 . 9 5 5} \\
\mathbf{p}=\mathbf{0 . 0 0 0}\end{array}$ & $\begin{array}{l}r=0.524 \\
p=0.002\end{array}$ & $\begin{array}{l}r=-0.138 \\
p=0.443\end{array}$ & $\begin{array}{l}r=0.004 \\
p=0.983\end{array}$ \\
\hline $\begin{array}{l}\text { Trunk FM } \\
\text { (kg) }\end{array}$ & & & & & & & - & $\begin{array}{l}r=0.804 \\
p=0.000\end{array}$ & $\begin{array}{l}r=0.595 \\
p=0.000\end{array}$ & $\begin{array}{l}r=-0.133 \\
p=0.460\end{array}$ & $\begin{array}{l}r=0.022 \\
p=0.904\end{array}$ \\
\hline $\begin{array}{l}\text { Peripheral } \\
\text { FM (kg) }\end{array}$ & & & & & & & & - & $\begin{array}{l}r=0.603 \\
p=0.000\end{array}$ & $\begin{array}{l}r=-0.126 \\
p=0.486\end{array}$ & $\begin{array}{l}r=-0.011 \\
p=0.949\end{array}$ \\
\hline $\begin{array}{l}\text { REE/FFM } \\
\text { (kcal/day/kg) }\end{array}$ & & & & & & & & & - & $\begin{array}{l}r=-0.092 \\
p=0.612\end{array}$ & $\begin{array}{l}r=-0.191 \\
p=0.286\end{array}$ \\
\hline $\begin{array}{l}\text { L1-L4 BMD } \\
(\mathrm{g} / \mathrm{cm} 2)\end{array}$ & & & & & & & & & & - & $\begin{array}{l}r=0.902 \\
p=0.000\end{array}$ \\
\hline
\end{tabular}

Adipon, adiponectin; EIG, urinary estrone 3-glucuronide; AUC, area under the curve; TT3, total triiodothyronine; FM, fat mass;

Periph, peripheral; REE, resting energy expenditure; FFM, fat free mass; BMD, bone mineral density.

* Using pooled data (all women, $\mathrm{n}=34$ ). Significant correlations are bolded. 
Table 4. Regression analysis (stepwise regression) of predictors of adiponectin*, estrogen exposure, and bone mineral density.

\begin{tabular}{cccccccc}
\hline & $\boldsymbol{B}$ & SE $\boldsymbol{B}$ & $\boldsymbol{\beta}$ & $\boldsymbol{F}$ ratio & $\boldsymbol{R}^{2}$ & $\boldsymbol{R}^{2}$ adj & $\boldsymbol{P}$ \\
\hline $\begin{array}{c}\text { Adiponectin*/FFM } \\
\text { Intercept }\end{array}$ & 3.273 & 0.093 & & & & & \\
Trunk Fat Mass & -0.065 & 0.013 & -0.657 & 23.538 & 0.432 & 0.413 & $<0.001$ \\
& & & & & & & \\
Estrogen Exposure & & & & & & & \\
Intercept & -10138.32 & 3769.86 & & & & & \\
TT3 & 5920.38 & 1883.09 & 0.504 & 9.885 & 0.254 & 0.228 & 0.004 \\
& & & & & & & \\
Total BMD & 1.803 & 0.227 & & & & & \\
Intercept & -0.161 & 0.057 & -0.464 & 7.948 & 0.215 & 0.188 & 0.009 \\
Adiponectin* & & & & & & & \\
L1-L4 BMD & & & & & & & \\
Intercept & 2.728 & 0.414 & & & & & \\
Adiponectin* & -0.406 & 0.104 & -0.565 & 13.683 & 0.321 & 0.297 & 0.001 \\
E1G AUC & 0.048 & 0.000 & 0.304 & 9.856 & 0.413 & 0.374 & 0.045 \\
\hline
\end{tabular}

All women, $\mathrm{n}=34$.

* signifies log adjusted data 
Figure 1

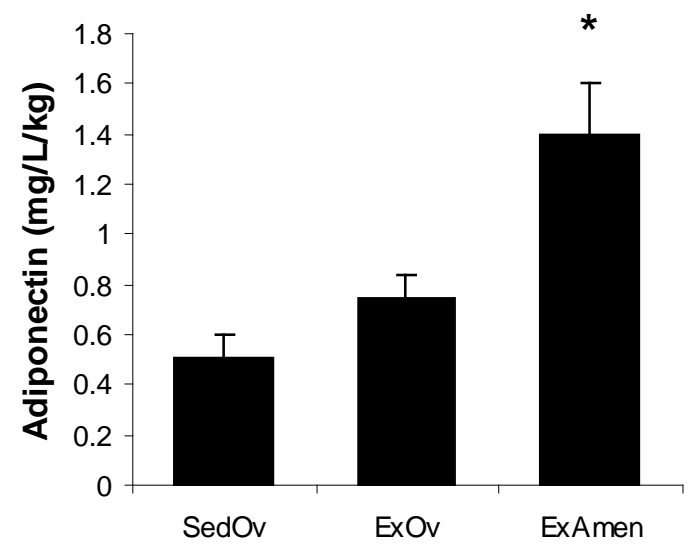

Menstrual and Ovulatory Status 
Figure 2

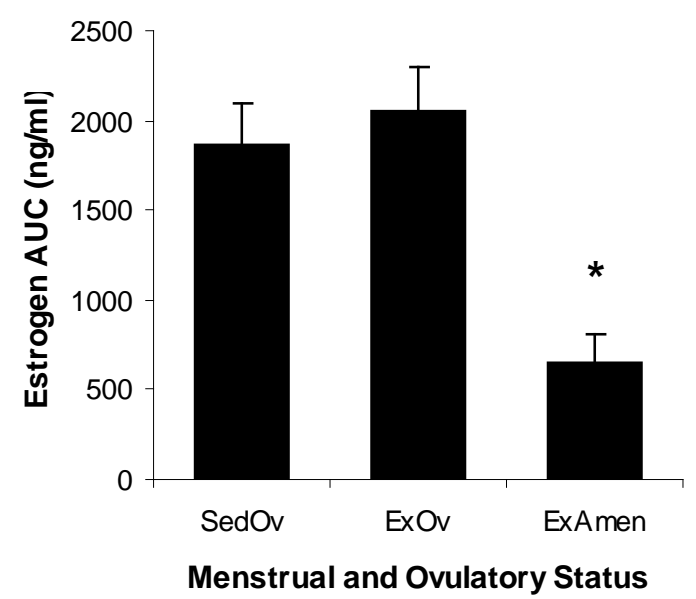


Figure 3

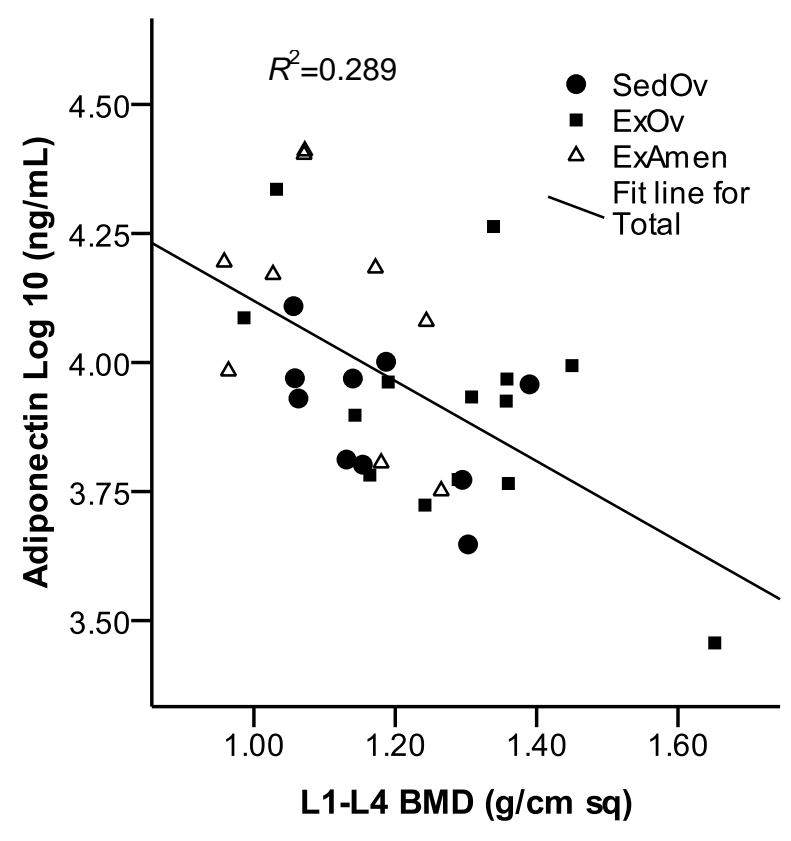

